\title{
SYMPOSIUM
}

\section{The impact of the phase of an epidemic of sexually transmitted infection on the evolution of the organism}

\section{K M E Turner, G P Garnett}

The evolution of any sexually transmitted organism will be influenced by prevailing epidemiological interactions. The optimum strategy for an organism to overcome treatment, either through drug resistance or cryptic infections, depends upon whether the method for identifying patients is passive (treating symptoms alone favours asymptomatic organisms) or active (screening favours resistant organisms). The use of mathematical models of competing strains of infection allows theoretical predictions for the outcome of evolution under a range of assumptions about potential phenotypes. The course of pathogen evolution has implications for the success of interventions, but the predictions presented need to be tested at the level of the community in carefully monitored interventions.

See end of article for authors' affiliations

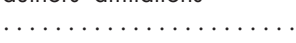

Correspondence to: Katherine Turner, Department of Infectious Disease Epidemiology, Faculty of Medicine, Imperial College of Science, Technology and Medicine, St Mary's Campus, Norfolk Place, London W2 IPG, UK; katherine.turner@ic.ac.uk

Accepted 29 November 2001 ally transmitted infections is the consequence of evolution. For any infectious organism this evolution is profoundly influenced by the epidemiological factors that constrain its environment. The organisms within an infection interact with one another and with the individual host. Likewise, within the host population, infections compete, altering their reproductive success.

For any organism, evolution is shaped by the mechanisms that introduce variation into the genome, the ways in which genes spread through populations, and the challenges which limit populations and thereby the success of different genes. The sequence of bases in DNA and RNA encoding the regulation and synthesis of enzymes and structural molecules is the raw material for evolution. On replication, molecular mechanisms of base substitution and deletion, or recombination and reassortment, configure this genetic raw material. Then, selection and neutral drift act to determine the distribution of different sequences in future generations. Chances for genetic recombination, genetic drift, and natural selection are a function of the population dynamics of organisms and, for infectious diseases, population dynamics is the main focus of epidemiology. The phase of an epidemic plays many roles in controlling evolutionary pressures, which will be explored here in the context of sexually transmitted infections (STIS).

As described elsewhere, an epidemic can be divided into five phases characterising growth and decline of a population of infectious organisms. $^{12}$ These are: phase I, the growth t is axiomatic that the current diversity of sexu- period, as the invasion of a host population occurs; phase II, the peak of the epidemic and hyperendemic phase when no controls have yet been imposed; phase III, a decline phase as controls are introduced and take effect; phase IV, a new endemic phase-that is, the steady state prevalence and incidence when controls are acting; and phase V, elimination, and potentially eradication. These phases could be applied to the global or local spread of an infectious organism.

By definition, STIs are caused by pathogens, whose evolution is intimately tied up with the diseases they induce, their ability to spread in new hosts, and their response to immunity and treatment. It is inevitable that when we consider the relation between the evolution and epidemiology of STIs we will be concerned with the phenotypes of the organisms (the actual expression of the characteristics encoded by gene which is influenced by both genes and the environment) and how these phenotypes influence their reproductive success. We can think of the pressures imposed on the sexually transmitted infection at each epidemiological phase in turn.

The founder effect will be important in phase I when an infection enters a host population and spreads with little competition. ${ }^{3}$ The founder effect is defined as the establishment of a new population by a few original founders, which carry only a fraction of the genotypes of the parental population. ${ }^{4}$ Particular genotypes will become common because they were present when the STI was introduced into the population. With small local outbreaks, as are currently observed in industrialised countries for bacterial STIs, there will be repeated episodes where founder infections become common in particular locations causing fluctuations in the common genotypes within a community, country, or region. Likewise, for infections within the host those organisms present in the infecting dose may be a limited subset of the infectious population allowing for random shifts in the genes represented in the pathogen population.

At a hyperendemic steady state (phase II), saturation of the infection in the population has occurred, when pre-emptive infection (or acquired immunity) reduces the reproductive success of each infection. It is in this phase that the transmissibility of the infection and the ability to persist in the individual host, avoiding acquired or innate immunity, would be optimised. This will generate and hone mechanisms to avoid the immune defences of the individual host and the host population. Examples of these mechanisms are antigenic variation as observed in gonorrhoea, chlamydia, and HIV, ${ }^{5-7}$ low levels of exposed antigens as appears to be the case for syphilis, ${ }^{8}$ latent 
state in an immune privileged site with continual reactivation as in the case of herpes simplex virus 2 (HSV-2), ${ }^{9}$ or minimal contact with systemic immunity, as appears to be the case with minimally invasive infections like human papilloma virus (HPV). ${ }^{10}$ Competition at this endemic prevalence may be particularly intense, especially if there is a group with a high risk of acquiring and transmitting infection, as is often the case for STIs, where continual re-exposure can occur for some individuals. The ability to invade an already infected host becomes a selective advantage in such circumstances, but concomitantly it becomes an advantage for the original infection to prevent further infection.

The key measure for evolutionary fitness is the reproductive success of the organism - that is, its representation in future generations. Normally we measure the reproductive success (the number of viable offspring) of the individual organism or gene. However, in infectious disease epidemiology we can also think in terms of the reproductive success of the infection, a colony of related individuals, and the reproductive number $\left(R_{t}\right)^{3}$ The basic reproductive number $\left(R_{0}\right)$ is defined as the average number of new infections arising from one index case in an entirely susceptible population. The value of the reproductive number at a given time $t\left(R_{t}\right)$ determines whether the related organisms are increasing $\left(R_{t}>1\right)$ or decreasing $\left(R_{t}<1\right)$ their representation at that time. The basic reproductive number, $\mathrm{R}_{0}$, applies only in the absence of density dependent constraints-in phase I, the invasion of a susceptible population. The influences on the reproductive number, in addition to the proportion of the host population susceptible, consist of three main elements: the duration of infectiousness, the pattern of contact within the host population, and the likelihood of transmission on contact. An increase in duration of infectiousness or transmissibility would increase the fitness of a pathogen.

While it is possible to hypothesise about optimum evolutionary strategies, the early academic consensus that parasites should evolve to become benign illustrates the potential pitfalls of such speculation. It was assumed that increasing the longevity of the host would also improve the fitness of parasites by maximising the parasites' own survival. ${ }^{11}$ However, this fails to take into account the potential correlation between pathogenesis and virulence. (Virulence has been defined differently across disciplines. To the clinical microbiologist, virulence is the ability to invade the host, causing disease; to the evolutionary biologist, virulence is a measure of the organism's ability to replicate.)

A trade-off is likely between the duration of infection and infectiousness, which may be associated with severity of disease. ${ }^{12-14}$ Biological explanations for the link between virulence and pathogenicity have been explored and may include increased replication, which increases pathogenicity but also increases fitness in terms of the ability to withstand host immune defences, and increased rates of transmission. ${ }^{15}{ }^{16}$ The level of disease associated with an infection will depend upon this trade-off and the biological mechanisms damaging the host.

Support for the earlier view was invoked from the severe symptoms and high mortality observed for many emerging infections, which then decline as the epidemic progresses. The first recorded outbreaks of syphilis, caused by Treponema pallidum, in the 15th century involved severe symptoms and rapid progression to death. ${ }^{17}$ Subsequently, disease progression has slowed and symptoms reduced in intensity. ${ }^{18}$ The myxoma virus strains, which cause myxomatosis in rabbits, were released in Australia in 1950. The initial virus was associated with a $90 \%$ fatality rate, but was soon supplanted by strains with a lower fatality rate. ${ }^{12}$ In both cases an intermediate level of virulence was maintained as the optimum in the trade of between transmission and survival.

In the later phases of an epidemic (III, IV, and V) following the introduction of interventions, very different selective pres- sures will apply. The characteristics of an infection that allow it to be targeted by an intervention become more significant, and will be selected against. In the case of treatment, this selective pressure could be for drug resistance or to avoid being identified (that is, selection against the characteristics that identify cases for treatment). Under the selective pressures of an intervention, new optimum characteristics are selected for, and the most successful organisms are likely to have a different phenotype from those most successful in phase II. Selection of traits such as drug resistance or asymptomatic infections could compromise the organism's ability to exploit its host, avoid immunity, or be transmitted. For example, in several organisms-including HIV, Escherichia coli, and Salmonella typhimurium - drug resistance is associated with a reduced rate of replication for some mutations; however, compensatory mechanisms have been shown to evolve rapidly in antimicrobial resistant viral and bacterial strains. ${ }^{20}{ }^{21}$ If the new organisms can compensate for the costs associated with resistance or asymptomatic infection then they will reestablish the phase II prevalence, causing the failure of the intervention. However, it is likely that resistant or asymptomatic infections are less transmissible and the new steady state prevalence will be lower than that of phase II. If the selection imposed by the intervention is sufficiently strong it will drive the population of pathogens to extinction. This is the "cost of selection" defined by Haldane, ${ }^{22}$ which limits the rate of change imposed by natural selection. The greater this cost the faster a sensitive gene will be eliminated and a resistant gene will dominate, but also the greater the chances of the entire population being eliminated.

In the last phase, phase $\mathrm{V}$, small isolated populations of organisms are likely to be selected by the main traits related to their niche as pathogens and the interventions against them, but are also more likely to be susceptible to the fixation or loss of genes through genetic drift. Genetic or neutral drift is where there are random shifts in the frequency of genes and alterations to gene sequences that are selectively neutral (for example, synonymous changes in bases which do not alter the amino acids coded for) spread through populations. ${ }^{23}$

To illustrate the influence of phase of epidemic on STI evolution, we use a mathematical model of two interacting strains. The model is that used by Hethcote and Yorke ${ }^{24}$ to study gonorrhoea epidemiology, with the additional complexity derived from including two strains. We first explore the ability of a genotype to invade a naive population (phase I) and then to invade a population where infection is hyperendemic (phase II). We highlight the importance of both the basic reproductive number-which determines the relative success of strains in a naive population-and of causing new infections rapidly, thus outcompeting rivals. We compare the ability of organisms to coexist and compete when they are able to exclude one another and when there is restricted cross immunity. In the extreme case of no cross immunity, the two strains are entirely independent and play no role in one another's evolutionary success. We then explore the changed situation in phases III, IV, and V when interventions have been included. In particular, we illustrate the relative merits of drug resistance and asymptomatic infection as evolutionary strategies.

\section{METHODS}

We introduce two versions of a two-strain deterministic model of gonorrhoea in a heterosexual population divided into four activity classes. Partnerships in this type of model occur instantaneously. A small proportion of the population (2\%) is in the highest activity class, the majority $(60 \%)$ is in the lowest. For simplicity in balancing the supply and demand for sexual partnerships, both sexes are assumed to have the same distribution of activity. We vary the values of the transmission probability per sex partnership, or the duration of infection for 

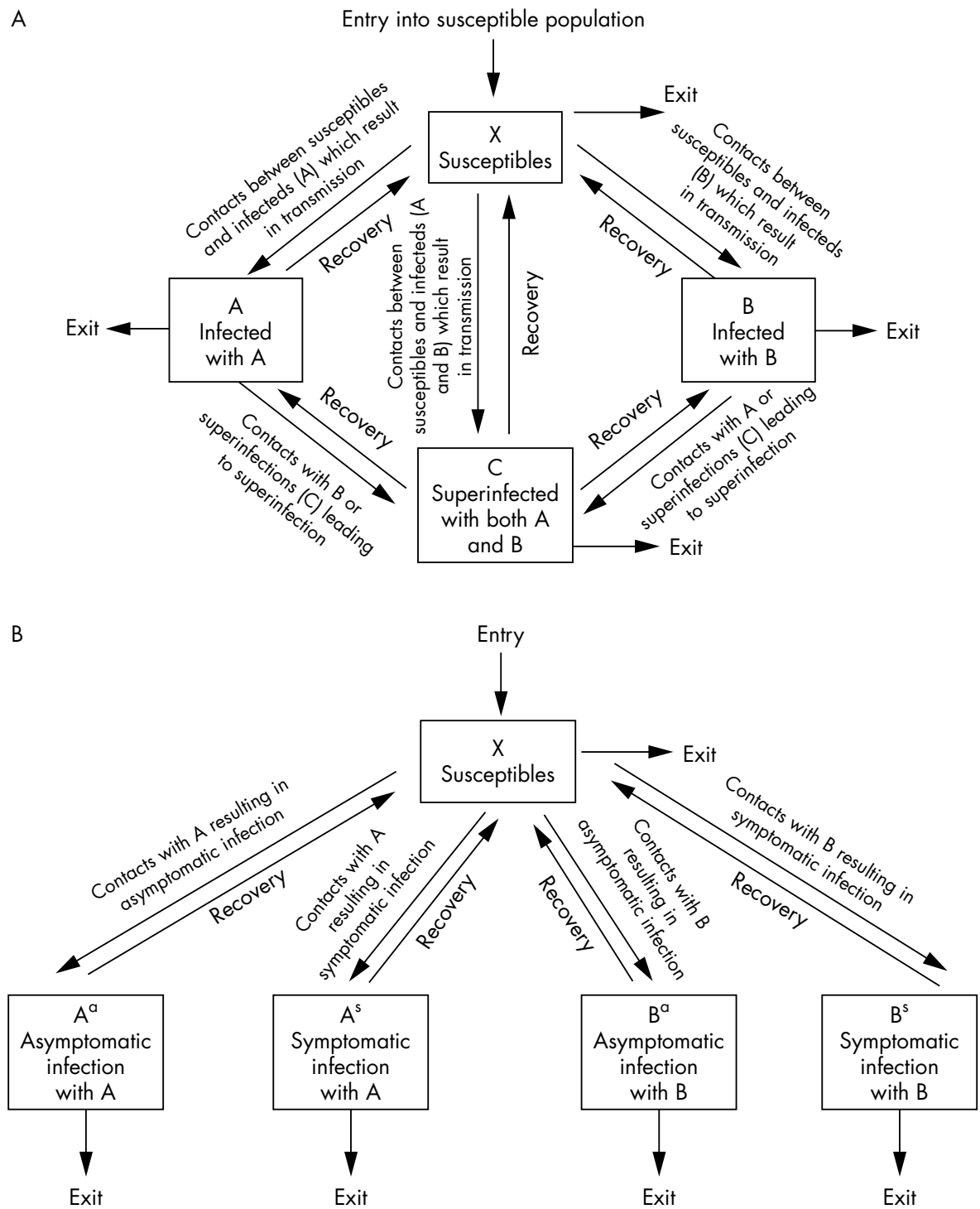

Figure 1 Flow diagrams describing the categorisation of infections in two strain models of gonorrhoea: $(\mathrm{A})$ model 1: a two strain model with superinfection; (B) model 2: a two strain model incorporating asymptomatic and symptomatic infection.

each strain, to represent different phenotypes. For example, resistance to antimicrobial treatment would generate a longer duration of infection compared with sensitive infections, when treatment is used. For all infections we assume that there is no acquired immunity, so recovery is to the susceptible class. In the case of dual infection, recovery may be from either or both strains.

Both models allow the parameter values describing the characteristics of strains to change during the course of the simulation. This could be to add a new strain or to represent the introduction of a treatment strategy. The impact of treatment is modelled by assigning new values for the duration of infections of the strains. The new values depend on the assumed sensitivity of the particular strain to treatment and whether the infection can be detected and treated (for example, asymptomatic infections may go untreated for a long period of time). The impact of these changes can then be seen in the context of different phases of the epidemic, depending when the switch is made.

The models are illustrated in fig 1 where the flow of people between categories is shown. In the first model, model 1 (fig lA) "superinfection" is possible and the host population falls into four categories: susceptible; infected with strain A; infected with strain B; and infected with both strains. Infection with one strain may confer some immunity to infection against the other, the strength of this cross protection varying from absolute (no superinfection) to negligible, where the strains are independent. Different strains could vary in their ability to inhibit rival strains-for example, by faster growth in vivo-but in the following examples cross protection is assumed to be symmetrical.

The second model, model 2 , is a special case, with total cross immunity, which includes the additional complexity of asymptomatic and symptomatic infections being separately represented and strains having an associated propensity to generate symptoms (fig 1B). Asymptomatic infections are widely assumed to be less transmissible than symptomatic infections-for example, because of a lower intensity of infection or a lower replication rate. However, it may also be the case that symptomatic infections are transmitted less often owing to increases in treatment seeking behaviour, decreases in sexual contacts, or increases in condom use. There is no good quantitative evidence to indicate which of these factors is most important, and the balance between the two conflicting assumptions is likely to vary in different populations with differing access to education and healthcare. A term has been 
included in the model to allow the transmissibility of asymptomatic infections to be modified (either increased or decreased) relative to symptomatic infections, depending on available empirical evidence, but we have not used this term in the examples given here.

Model 2 has five categories: susceptible (X); asymptomatic infections with strain $\mathrm{A}\left(\mathrm{A}^{\mathrm{a}}\right)$; symptomatic infections with strain $A\left(A^{s}\right)$; asymptomatic infections with strain $B\left(B^{a}\right)$; and symptomatic infections with strain $B\left(B^{s}\right)$. The probability that when an infection occurs it remains asymptomatic differs between the strains (strain A: 80\% asymptomatic and 20\% symptomatic; strain B: $40 \%$ asymptomatic and $60 \%$ symptomatic infections). Mathematical descriptions of the models and the parameters used are given in the appendix.

\section{RESULTS}

We can use theory, as illustrated by model results, to predict the result of competition between strains during the initial spread and saturation of infection (phase I and II).

First, if there is cross protection the outcome of competition between strains will be determined by their relative $\mathrm{R}_{0}$. The strain with the higher $\mathrm{R}_{0}$ will dominate and drive other strains to extinction. Recent studies of HIV, for example, suggest that HIV- 1 will eventually competitively exclude HIV- $2 .{ }^{25}$ This general rule of competitive exclusion with a higher $\mathrm{R}_{0}$ applies for a deterministic large population approximation, when there is total cross immunity. However, it is possible that despite a lower basic reproductive number, a strain with a higher transmissibility (and by implication shorter duration of infectiousness) could spread first, only to eventually be overtaken by the strain with a higher basic reproductive number associated with a lower transmissibility but longer duration-which could persist at an extremely low prevalence early in the epidemic. In smaller populations the first strain drives another strain to extinction before the overall reproductive number is made to count. If two strains have the same basic reproductive number, which is likely if they are genetically very similar, they will coexist. However, a strain introduced when another is already endemic can only invade if it has a higher $\mathrm{R}_{0}$ than the existing strain.

Figure 2 illustrates the importance of transmissibility (in model 1), during the initial phase of the epidemic (total cross immunity), with the course of epidemics illustrated in fig 2A, and the equilibrium prevalences in fig $2 \mathrm{~B}$. Both strains have the same $\mathrm{R}_{0}$ (to permit coexistence) but different transmission parameters. The transmissibility of strain B is increased and, in compensation, its duration of infection is decreased, while parameters for strain A were held constant. When the strains are identical in transmissibility and reproductive number they cause the same numbers of cases. When B is less transmissible the initial increase of strain B is slower and it ultimately causes fewer cases. This is reversed as the transmissibility of B exceeds that of A. The total number of infections is the same in each case because the $\mathrm{R}_{0}$ of both strains is constant. High transmissibility in the growth phase enables a strain to establish itself in the available ecological niche quickly and is a selective advantage.

The results described above apply to mutually exclusive strains, but it is quite possible that multiple strains coexist within infections. We explore the simplest case of two strains and call infection with more than one strain "superinfection". The evolutionary outcome of superinfection will depend upon which organisms are transmitted to contacts and when recovery occurs. Superinfection can occur in two ways-by serial acquisition (that is, acquisition of one strain followed by the separate acquisition of another strain), or by the concurrent transmission of both strains from a superinfected individual. Both are biologically feasible and may lead to different outcomes in terms of competition between the two strains. Serial transmission may present more difficulties for the
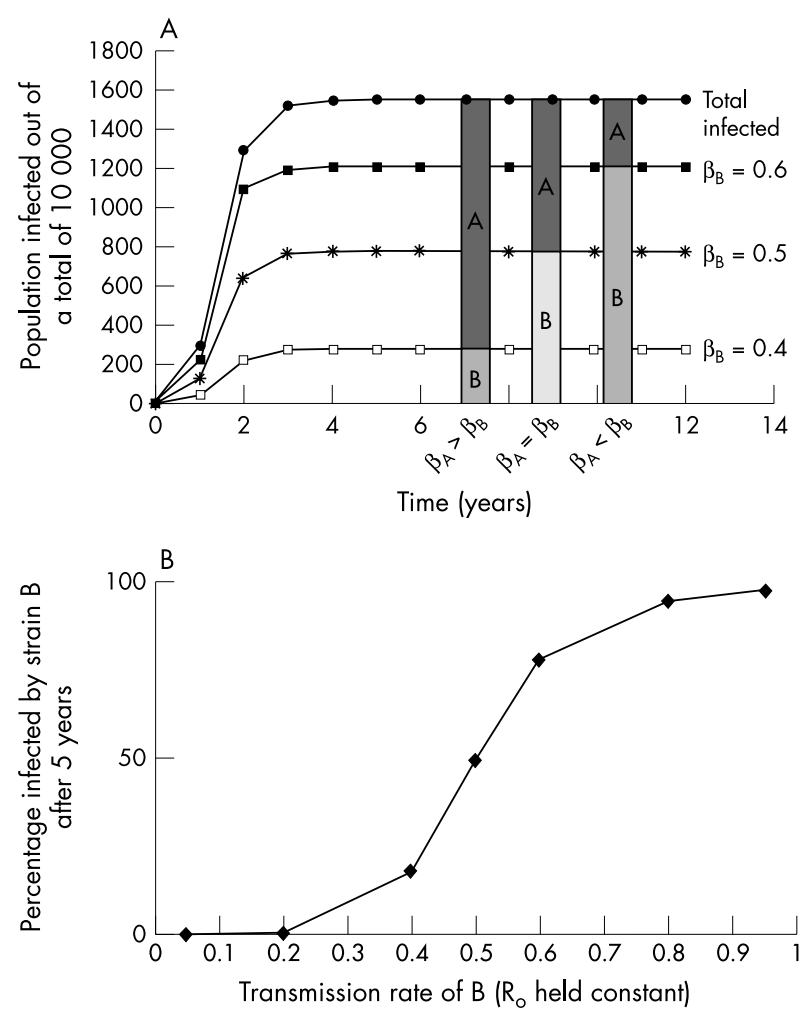

Figure 2 The outcome of spread of two strains in the absence of superinfection with the same basic reproductive number for each strain but variation in the transmission coefficient of strain B. (A) The time course of the epidemic as a whole and the epidemic of strain B. The bars show the equilibrium prevalence of the two strains. (B) The equilibrium percentage of strains that are $B$ as a function of its transmission probability. $\left(\beta_{A}=0.5, \sigma_{A}=2 ; \beta_{B}=0.1, \ldots, 0.9, \sigma_{B}=\right.$ $0.3, \ldots, 3.62)$.

invading pathogen, as it not only has to overcome the host defences but also has to establish itself in the face of competition from the resident strain. To transmit to a preinfected individual, a strain may need to have a higher replication rate or occupy a different ecological niche, which avoids direct competition in the host. Alternatively, preinfection may actually aid further infections by altering the host environment to be more hospitable to invading bacteria, for example by changing the $\mathrm{pH}$ or causing inflammation. Different strains might conceivably have differing success in male and female hosts, which could allow both to persist. The order of serial infection may be important, with a hierarchy of invasiveness of different strains. However, in the current model we do not pursue this level of complexity.

The time scale over which multiple infecting organisms coexist is important. If a strain can outcompete a rival before the next opportunity for transmission, then superinfections will be less important in the overall prevalence of infections. Instead there would be a hierarchy of replacement and direct competition between strains, where ability to dislodge a rival from a host would play a role in the success of competing strains. Assuming that superinfection lasts long enough to be transmitted directly to another individual, what actually happens within the next infected individual will be the result of competition between the strains for resources.

Another important interaction, which gonococci in particular are capable of, is the exchange of genetic material, potentially resulting in the evolution of novel strains in a short space of time. $N$ gonorrhoeae are competent for gene transfer or plasmid exchange all the time, so it seems highly likely that this is an important mechanism for this organism. In these models, 


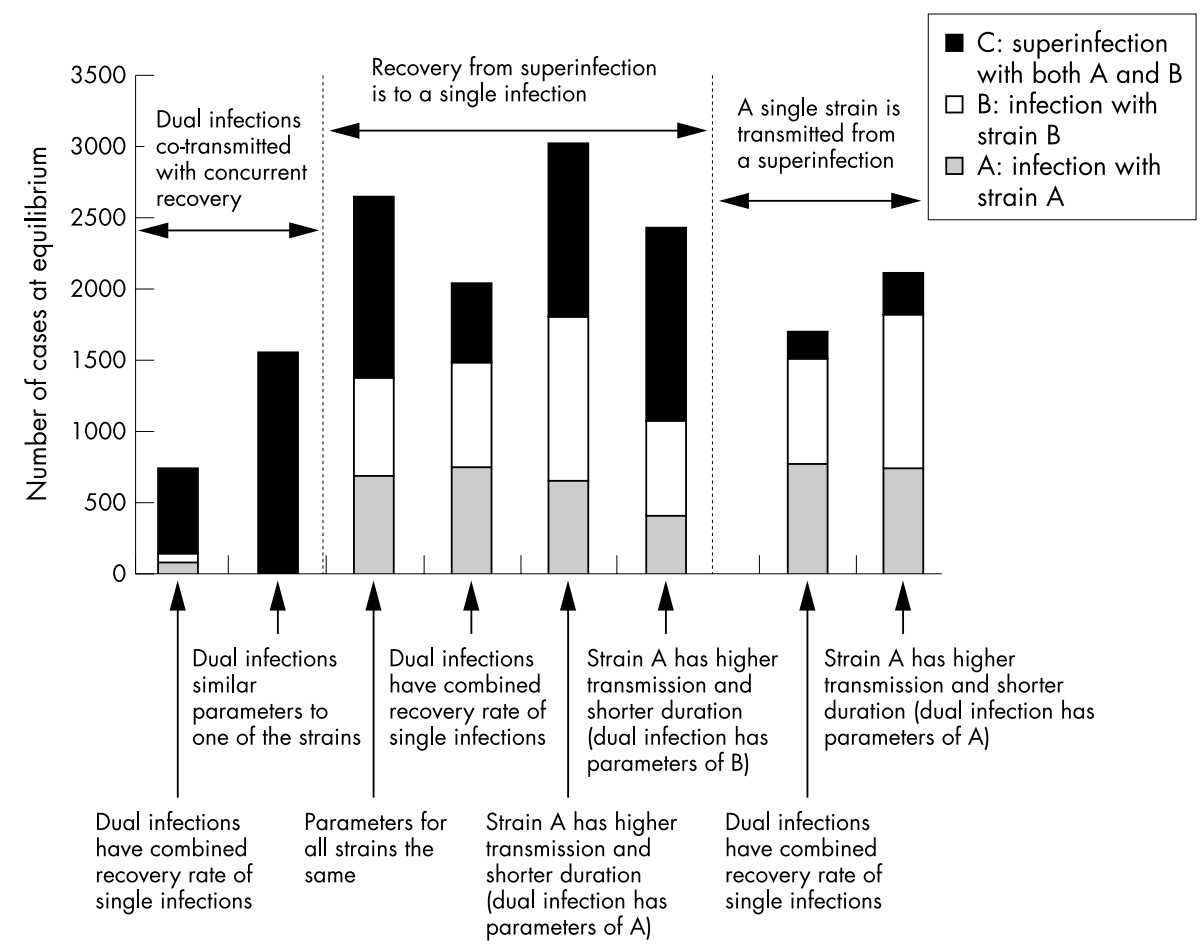

Figure 3 The equilibrium prevalence of individual infections and coinfection with two strains in model 2 defined in the appendix, for a range of assumptions about the biology of superinfection. Intermediate cross immunity $\phi_{A}=\phi_{B}=0.5$ throughout. Transmission: either $\beta_{A}=\beta_{B}=0.5$ or $\beta_{A}>\beta_{B}$ $\left(\beta_{A}=0.5, \beta_{B}=0.2\right)$, and $\beta_{C}=\beta_{A}$ or $\beta_{C}=\beta_{B}$. Recovery: either $\sigma_{A}=\sigma_{B}=0.5$ or $\sigma_{A}>\sigma_{B}\left(\sigma_{A}=2, \sigma_{B}=0.788\right)$ (greater recovery rate implies shorter duration). Transmission of both strains from superinfection: $\left(\gamma_{A}=0\right)$, except last two columns $\left(\gamma_{A}=0.5\right)$.

however, we have assumed that strains remain distinct during superinfection.

Recovery from superinfection may be faster than from single infections-for example, owing to a greater host immune response or increased likelihood of symptoms and hence treatment seeking behaviour. Alternatively, superinfections may be more difficult for the host to clear, as in the case of increased replication by the competing strains. Recovery from superinfection may be to the susceptible class, for example if effective treatment is obtained, or to single infection with either strain, as when competition between strains removes one strain, or if strains have different sensitivities to antimicrobial treatment. The recovery rate from superinfection may be faster or slower than for single infections and may be dependent on the recovery rates of the individual strains present.

If cross immunity is relaxed and superinfection can occur, various outcomes are possible, depending on the selective pressures acting on the system and the characteristics of the two competing strains..$^{25}{ }^{26}$ The equilibrium prevalence of the two strains is explored in fig 3, where there is only a moderate level of cross protection so that coexistence of strains in the population will be frequent.

We explore three sets of possibilities. (1) Both strains are transmitted concomitantly from a superinfection, and recovery is from both strains simultaneously-for example, all recovery is due to treatment and both strains are equally susceptible. In this case superinfection becomes the sole form of infection despite cross protection unless it causes more rapid recovery, giving an advantage to single strain infections. (2) Both strains are transmitted together but recovery from superinfection involves the removal of one strain before recovery from the other strain becomes possible. For example, this could be due to within-host competition, where one strain rapidly drives the other to extinction and there is some kind of hierarchy of invasiveness, or alternatively there is a differential response to treatment. Here superinfection can increase the average duration of infections, and the relative recovery rates of single and dual infections determine their relative frequency, with a longer duration of infection for a strain providing a greater advantage than a comparable higher transmissibility. (3) Transmission from a superinfected host always generates a single infection. This could be due to stochastic effects or differences in transmissibility of the two strains. Here superinfection is much less common.

Stronger cross protection decreases the overall prevalence of infection (with no cross protection, no potential infections are prevented). With no superinfection, an invading strain may only invade if its $\mathrm{R}_{0}$ is higher than the existing strain. Superinfection relaxes the impact of phase of an epidemic on the outcome of competition between strains, as the timing of a strain entering the population no longer determines its relative success. Additionally, a strain with a lower $\mathrm{R}_{0}$ may invade and coexist with the more infectious strain. The relative proportions are determined by the $\mathrm{R}_{0}$ of the two strains, and with some cross protection the relative duration of infection is the critical factor because long infections prevent proportionately more infections with the opposite strain than short ones.

\section{THE INTRODUCTION OF TREATMENT}

The implementation of effective treatments or changes in host behaviour imposes new selective pressures in the ongoing competition between host and parasite. This may select for novel traits, such as resistance, or for increases in the likelihood of asymptomatic infection. The success of different evolutionary strategies for the pathogen depends upon a trade-off between the costs and benefits of the phenotypic change. Possible evolutionary strategies are constrained by the mechanisms available to the organism from within its own gene pool or drawn from other organisms through recombination or the transfer of plasmids. ${ }^{27}$ Because of rapid replication and various genetic mechanisms (for example, DNA transfer), the evolution of bacteria can be extremely rapid. If competition between strains for hosts is intense, small advantages in reproductive fitness can quickly translate to large changes in 
frequency distributions of different strains. ${ }^{1428}$ Frequent population bottlenecks-as may occur during transmission to a new host and with bacterial population expansion in a host-provide fertile conditions for the rapid evolution and distribution of new strains.

In phases III/IV of an epidemic, the implementation of controls exerts a strong selective pressure on the bacterial populations, resulting in the evolution of novel strains. Treatment of symptomatic gonorrhoeal infections has led to the evolution of resistant strains. For example, Neisseria gonorrhoea strains resistant to penicillin were isolated 30 years after the introduction of the drug. ${ }^{29}$ Following the introduction of other antimicrobial compounds new resistant strains have appeared, for example to tetracycline ${ }^{30}$ and fluoroquinolones. ${ }^{31}$ There has been general theoretical work modelling the evolution and spread of resistance in bacteria which has concentrated on non-specific consumption of antibiotics and nosocomial infections. ${ }^{32-34}$ Here we model the impact of treatment on two competing strains.

Figure 4 illustrates the impact of treatment on two competing strains, with no superinfection. In fig 4A before treatment, strains A and B both have the same parameters and so each contributes $50 \%$ of total prevalence. Strain A is sensitive and strain B resistant to treatment. When treatment is implemented, the recovery rate of the susceptible strain A is greatly increased, lowering its effective reproductive number. Strain B rapidly outcompetes $\mathrm{A}$ and drives it to extinction. If treatment reduces the reproductive number of $A$ to less than 1 it will become extinct, whether or not there is competition from another strain. Even if the basic reproductive number $\left(R_{0}\right)$ has not been reduced to less than 1 , competition between strains can drive the sensitive strain A from the population when there is complete cross protection. Despite the reduction in prevalence of the sensitive strain the total prevalence of gonorrhoea is eventually restored because the resistant strain is released from competition.

The effect of resistant infection being introduced rather than being common at the outset is illustrated in fig 4B. Again the reproductive numbers of $\mathrm{A}$ and $\mathrm{B}$ are identical in the absence of treatment but $\mathrm{A}$ is sensitive to treatment and $\mathrm{B}$ is resistant. In the absence of treatment, strain B would not be able to invade. Two alternatives are illustrated-first, when treatment has no effect on B it invades and rapidly spreads, replacing A and re-establishing prevalence; second, when treatment has some effect on strain B (for example, with retreatment after initial treatment failure). Here $\mathrm{B}$ is still able to invade, but somewhat more slowly and with a lower final prevalence because of a lower effective reproductive number. Which alternative is most likely depends upon the mechanism of resistance, the other costs it imposes, and the availability of second line drugs.

Resistant strains are able rapidly to outcompete sensitive strains when there is no superinfection owing to the introduction of a significant difference in the effective reproductive number. Without superinfection, two strains can only coexist if they have the same $\mathrm{R}_{0}$. When superinfection is permitted, the situation becomes more complicated and is discussed in detail elsewhere. ${ }^{25}{ }^{26}$ Competition between strains is no longer absolute, and strains with different basic reproductive numbers can coexist in a host population.

During phases III, IV, and V, in the presence of treatment asymptomatic infections may play an extremely important role in determining the success of a strain. We examine the effect of different treatment strategies on competition between strains with different asymptomatic and resistant phenotypes using model 2 .

In fig 5 both strains have the same basic reproductive number before treatment and the same transmission/duration parameters, but strain A generates a higher proportion of asymptomatic infections (80:20, asymptomatic:symptomatic) compared with B (40:60). For the purposes of this discussion,
A

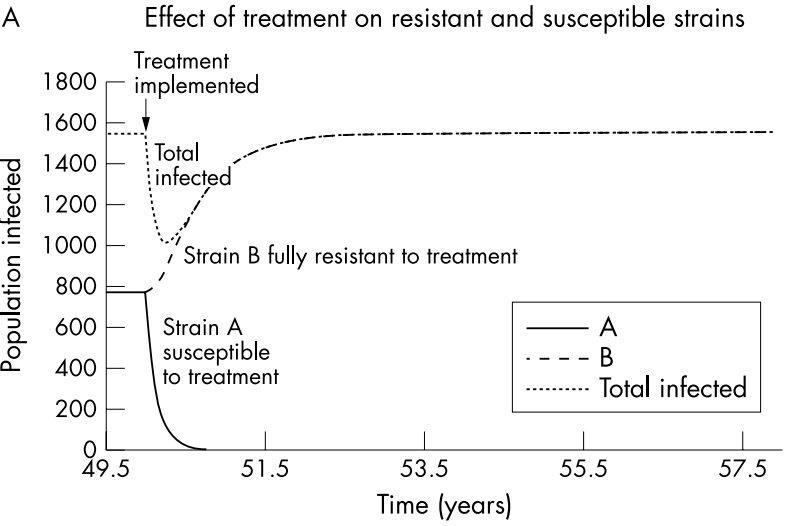

B

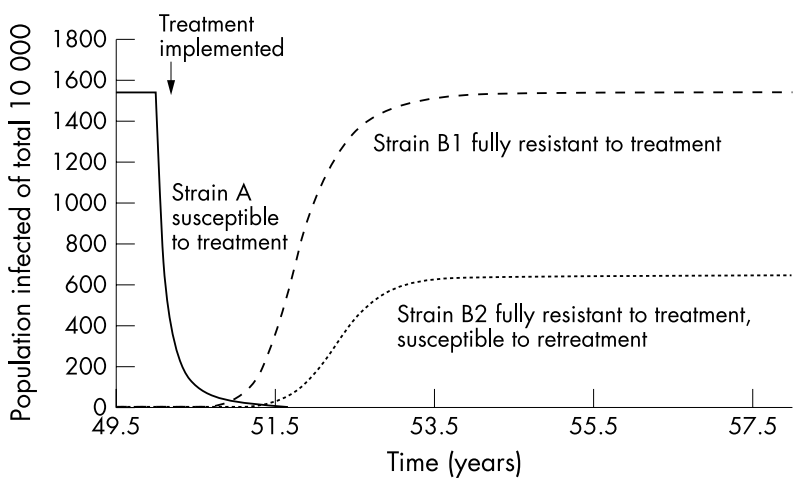

$-\mathrm{A}$

- - - B Total resistance to treatment

........ B Retreatment after initial treatment failure

Figure 4 Impact of treatment on resistant and susceptible strains during phases III and IV of an epidemic in the absence of superinfection (that is, total cross immunity). (A) The resistant stain is present when infection is introduced. Before treatment: $R_{0} A=R_{0} B$; transmissibility (per contact) $\beta_{A}=\beta_{B}=0.5$; recovery (individuals per year) $\sigma_{A}=\sigma_{B}=2$. After treatment: transmissibility $\beta_{A}=\beta_{B}=0.5$; recovery $\sigma_{A}=10, \sigma_{B}=2$. (B) Resistant strain (strain $B$ ) introduced with one infected individual of each sex at the same time as treatment. Before treatment: $R_{0} A=R_{0} B$; transmissibility (per contact) $\beta_{A}=\beta_{B}=0.5$; recovery (individuals per year) $\sigma_{A}=\sigma_{B}=2$. After treatment: transmissibility $\beta_{A}=0.5, \beta_{B}=0.5$; recovery $\sigma_{A}=10, \sigma_{B 1}=2, \sigma_{B 2}=4$.

we assume that the recovery rates - for a particular strain-of both asymptomatic and symptomatic infections are the same in the absence of treatment. The effect of treatment is incorporated in the model by decreasing the duration of infection of those treated, according to whether the strain is susceptible or resistant. Outcomes are shown two years after treatment, when levels have largely stabilised-that is, in phase IV of the epidemic, the endemic situation after controls are introduced.

Column (a) shows the distribution of infections by strain and by asymptomatic or symptomatic infection before treatment. As expected, each strain accounts for $50 \%$ of the total infections and infections are asymptomatic and symptomatic according to the ratios above. If symptomatic infections are treated and both strains are sensitive to drug (column b) there is a modest decrease in the overall prevalence. While symptomatic infections decrease quite dramatically, prevalence is maintained by asymptomatic infections. The proportion of prevalent infections that are asymptomatic increases for strain A to $95 \%$, and for strain B to $83 \%$. Strain A has a higher probability of causing asymptomatic infection and consequently a greater proportion of those infected are 


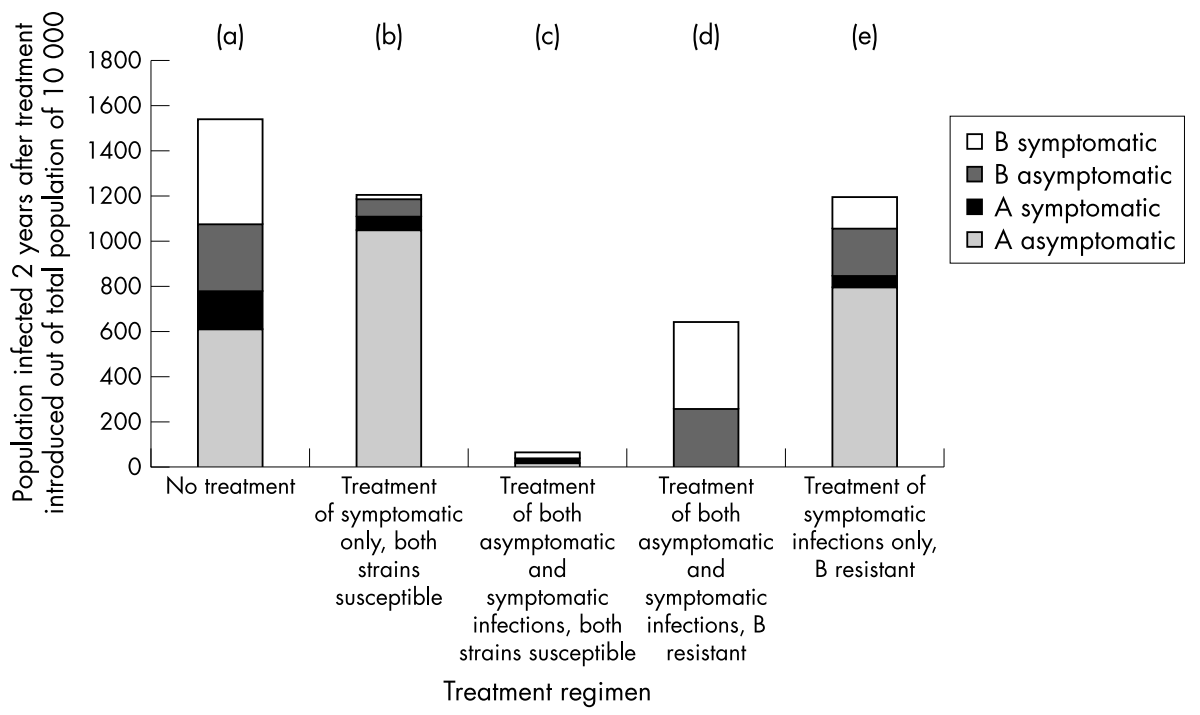

Figure 5 The impact of treatment targeted at symptomatic infections or all infections after 2 years on the prevalence of strains with different levels of resistance and propensities to cause asymptomatic infections. Column a, no treatment: transmissibility $\beta_{A}=\beta_{B}=0.5 ;$ recovery $\sigma_{A}=\sigma_{B}=2$. Column $b$, treatment of symptomatic infections: $\sigma_{A}=\sigma_{B}=10$. Column c, treatment of all infections: $\sigma_{A}=\sigma_{B}=8$. Column $d$, all infections treated: recovery rates of treated infections $\sigma_{A}=10, \sigma_{B}=4$. Column e, symptomatic infections only treated: recovery rates of treated infections $\sigma_{\mathrm{A}}=10$, $\sigma_{\mathrm{B}}=4$.

untreated and the proportion of all infections caused by A increases to over $90 \%$.

The impact of the introduction of a screening programme, or comparable strategy-for example contact tracing and partner notification-which results in the treatment of both asymptomatic and symptomatic infections is illustrated in column (c). If treatment decreases the basic reproductive number of strains A and B to less than 1, both will become extinct. If, however, the basic reproductive number for each strain remains above 1 , both infections can persist, retaining the same proportions of A to B and of asymptomatic to symptomatic infections, but at a much lower overall prevalence.

A similar scenario is illustrated in column (d), where both types of infection can be detected and treated, but strain A is sensitive and B resistant. Competition between A and B will quickly drive strain A to extinction because of the lower effective number. The recovery rate of the resistant strain B is still increased owing to retreatment with second line options, so its prevalence falls.

In column (e), as in column (b), symptomatic infections alone are treated, and strain $\mathrm{A}$ is sensitive and $\mathrm{B}$ resistant, but strain A is more likely to lead to asymptomatic infections. Both strains persist for at least two years, but the propensity to cause asymptomatic infections is a greater advantage than resistance, illustrating the impact that failure to identify and treat asymptomatic infections may have. Unless there is an effective screening programme to identify asymptomatic cases, surveillance data on the incidence of symptomatic gonorrhoea might show a dramatic decrease masking the continued high levels of asymptomatic infections. For columns (e) and (b) the incidence of symptomatic infections of both strains declines and eventually reaches a new equilibrium point, when strain A outcompetes B. The time taken to reach the new equilibrium is determined by the relative difference between the $\mathrm{R}_{0}$ of $\mathrm{A}$ and $\mathrm{B}$ after treatment. For these particular parameters, strain $A$ has a higher $R_{0}$ than $B$ after treatment and will eventually dominate, but for different proportions of asymptomatic infections for the strains or different responses to treatment, strain B would dominate. If the benefit conferred by resistance is equivalent to the benefit gained from avoiding treatment owing to a higher proportion of asymptomatic infections-that is, if the effective $\mathrm{R}_{0}$ values after treatment is introduced are the same-both strains can coexist indefi- nitely. If the $\mathrm{R}_{0}$ values are similar, then both strains may coexist for many years before one or other dominates.

Empirical evidence of asymptomatic strains gaining a selective advantage is scarce, probably because it has not been sought. In the case of gonorrhoea, where asymptomatic infections are common, particular strains may be associated with asymptomatic or very mild infection, for example the AHU auxotype. ${ }^{35}$ In Washington, the prevalence of the AHU auxotype has declined steadily from 52\% between 1971 and 1074 to $0 \%$ in 1994 to 1996 . This was believed to reflect increased screening efforts, resulting in treatment of more asymptomatic infections. More recently (1996 to 1997), the CU auxotype, which is also associated with asymptomatic infection, has increased in prevalence in this region, despite an overall decline in gonorrhoea rates of $80 \% .^{36}$ The exact reasons for the changes in subtype distribution of gonorrhoea are not well understood. This CU auxotype is more likely than other auxotypes to be misdiagnosed as non-gonococcal urethritis, which may account for part of the increase. Other possible explanations include decreased screening, decreased competition between strains, and genetic drift resulting from purely stochastic events facilitated by the lower population size and the random introduction and disappearance of subtypes.

\section{DISCUSSION}

The ecology and evolution of an infectious disease are influenced by both the natural dynamics of epidemics and the interventions used to control them. Using an extension of a mathematical model to include two competing strains, with the possibility of superinfection or asymptomatic infections, we have explored the outcome of competition to illustrate conditions leading to coexistence and the optimum phenotypes of organisms according to the phase of epidemic. In the early phases of an epidemic, where the majority of contacts are with susceptible hosts, the simplest models of competition suggest that transmissibility is a key attribute. If strains can coexist within a host, the impact of transmissibility on the success of competing strains is reduced and instead the duration of infection becomes paramount. Founder effects are also important at this stage, as once a strain is established in the population, only strains with a higher basic reproductive number $\left(\mathrm{R}_{0}\right)$ can invade. 
The biological mechanisms allowing co-infection and its clinical consequences are poorly specified. To generate testable predictions from models including superinfection, assumptions need to be based on the biology of specific organisms. For example, there is convincing evidence ${ }^{37}$ that mixed infections of gonococci do occur and that two strains can be transmitted simultaneously, depending on the relation between the course of infection and sexual exposure. However, competition between strains within the host is intense and it seems likely that one of the strains-or a novel composite descendant, resulting from gene exchange between different lineageswill rapidly come to dominate in a particular host and be transmitted onwards. It is likely that intense competition between strains in high risk (core) individuals may be important in driving the evolution of novel strains.

The impact of different treatment strategies on the evolution of STIs like gonorrhoea may differ at different phases of an epidemic. Early interventions, during the growth phase, are likely to have more impact than those made once a strain is endemic. The need for identifying and treating asymptomatic infections is highlighted. Treatment of symptomatic infections only has a small effect on the total prevalence of infections (depending on the proportion of asymptomatic infections), but reduces the number of symptomatic infections. The increase in the proportion of infections that are asymptomatic may result not from genetic changes in the population of organisms, but simply as a product of the asymptomatic infections not being curtailed in comparison with symptomatic infections. A decrease and subsequent stabilisation in the incidence of symptomatic cases is seen in the model (data not shown) when only symptomatic infections are treated; therefore, the observed decline and subsequent levelling off of reported cases (for example, in the USA ${ }^{38}$ ) could be in part explained by this effect. There will be a selective advantage to organisms that are prone to cause asymptomatic infections, which could well further increase the proportion of infections without obvious symptoms. A major concern would be that such infections still have serious long term sequelae. In the example shown, strains causing a high proportion of asymptomatic infections could compete well, even against strains resistant to antimicrobial treatment. For all cases it is clear that treatment will act as a strong selective force for novel strains. Advantages in resisting or avoiding treatment will result in the rapid spread of a strain through the population and elimination of competitors.

In our simulations we assume extreme levels of competition between strains, which in reality may not exist owing to heterogeneities in hosts or more subtle differences in the biological properties of the strains and their ability to colonise different hosts. The deterministic model assumes that partnerships are instantaneous and there is no concurrency. Therefore two features of real partnerships- that is, the duration of partnership and concurrency-which have been shown to affect the epidemiology of STIs ${ }^{39}$ are not captured in these models. Models that include more detail and have been validated are necessary to predict the outcome of treatment strategies and inform decision makers.

Only through careful monitoring of the genotype of organisms, the link between the genotype and the in vivo phenotype, and the relation to epidemic phase will it be possible to gain a full understanding of STD evolution. Such an understanding would underpin the construction of the detailed and well validated models necessary to predict the outcome of treatment strategies.

\section{ACKNOWLEDGEMENTS}

We would like to thank the reviewers, Stephen Moses and James Blanchard, for their thoughtful comments.
Authors' affiliations

K M E Turner, G P Garnett, Department of Infectious Disease

Epidemiology, Imperial College of Science, Technology and Medicine, St

Mary's Campus, London W2, UK

\section{APPENDIX}

Model 1. Gonorrhoea: two strains, two sexes, and four activity classes with the possibility of superinfection.

Subscripts refer to the sex and activity class. In the case of sex, $k=0$ for men, $k=1$ for women while $k^{\prime}$ refers to the opposite sex to $\mathrm{k}$, and for activity $l$ defines the class (from 1 to 4 ), according to average rate of partner change per year, $c_{k l}$. The superscripts $A, B$ associated with parameters identify the strain of the infection, and $C$ a dual infection. The subscripts stratify the state variables, which are as follows:

$\boldsymbol{X}_{k l}$ - population of susceptible individuals of sex $k$ and activity class $l$

$\boldsymbol{A}_{k l}$ - population of hosts infected with strain A of sex $k$, activity class $l$

$\boldsymbol{B}_{k l}$ - population of hosts infected with strain B of sex $k$, activity class $l$

$C_{k l}$ - population of hosts infected with strains A and B of sex k, activity class $l$

$\boldsymbol{N}_{\text {- }}$ - total population, sex $\mathrm{k}$, activity class

In order to simplify the equations we first define the force of infection $(\lambda)$ for each strain (A and B) and for coinfection. The force of infection is the product of the transmissibility of the strain $(\beta)$, the contact rate (c), and the proportion of the total population infected with the strain, contacts with whom are determined by the mixing matrix $\left(\rho_{\mathrm{klm}}\right)$. The force of infection is equivalent to the incidence rate per susceptible individual.

Thus for an individual of sex $\mathrm{k}$, activity class $\mathrm{l}$, the forces of infection for strains $\mathrm{A}$ or $\mathrm{B}$, or coinfection $\mathrm{C}$ are:

$$
\begin{aligned}
& \lambda_{k l}^{A}=c_{k l} \beta_{k}^{A} \sum_{m=1}^{4} \rho_{k l m}\left(\frac{A_{k^{\prime} m}}{N_{k^{\prime} m}}\right) \\
& \lambda_{k l}^{B}=c_{k l} \beta_{k}^{B} \sum_{m=1}^{4} \rho_{k l m}\left(\frac{B_{k^{\prime} m}}{N_{k^{\prime} m}}\right) \\
& \lambda_{k l}^{C}=c_{k l} \beta_{k}^{C} \sum_{m=1}^{4} \rho_{k l m}\left(\frac{C_{k^{\prime} m}}{N_{k^{\prime} m}}\right)
\end{aligned}
$$

The equations describing the model are as follows:

$$
\begin{aligned}
\frac{d X_{k l}}{d t}= & \mu N_{k l}-X_{k l}\left(\lambda_{k l}^{A}+\lambda_{k l}^{B}+\lambda_{k l}^{C}\right)+\sigma_{k}^{A} A_{k l} \\
& +\sigma_{k}^{B} B_{k l}+\sigma_{k}^{C}\left(1-\left(\omega^{A}+\omega^{B}\right)\right) C_{k l}-\mu X_{k l} \\
\frac{d A_{k l}}{d t}= & X_{k l}\left(\lambda_{k l}^{A}+\gamma^{A} \lambda_{k l}^{C}\right) \\
& -A_{k l} \phi^{A}\left(\lambda_{k l}^{B}+\left(1-\gamma^{A}\right) \lambda_{k l}^{C}\right)+\sigma_{k}^{C} \omega^{C} C_{k l} \\
& -\left(\sigma_{k}^{A}+\mu\right) A_{k l} \\
\frac{d B_{k l}}{d t}= & X_{k l}\left(\lambda_{k l}^{B}+\gamma^{B} \lambda_{k l}^{C}\right) \\
& -B_{k l} \phi^{B}\left(\lambda_{k l}^{A}+\left(1-\gamma^{B}\right) \lambda_{k l}^{C}\right)+\sigma_{k}^{C} \omega^{B} C_{k l} \\
& -\left(\sigma_{k}^{B}+\mu\right) B_{k l} \\
\frac{d C_{k l}}{d t}= & X_{k l}\left(1-\left(\gamma^{A}+\gamma^{B}\right)\right) \lambda_{k l}^{C} \\
& +A_{k l} \phi_{k}^{A}\left(\lambda_{k l}^{B}+\left(1-\gamma^{A}\right) \lambda_{k l}^{C}\right) \\
& +B_{k l} \phi_{k}^{B}\left(\lambda_{k l}^{A}+\left(1-\gamma^{B}\right) \lambda_{k l}^{C}\right)-\left(\sigma_{k}^{C}+\mu\right) C_{k l}
\end{aligned}
$$

Parameter definitions used in models (for two general strains, A and $\mathrm{B}$ ) are shown in table $\mathrm{l}$.

Mixing matrix definition. $\rho_{k l m}$ is a mixing matrix, the elements $\mathrm{klm}$ of which are the probability that an individual of sex $k$ in activity class $l$ will form a partnership with a member of activity class $m$ of the 


\begin{tabular}{|c|c|c|c|}
\hline Parameter & Definition & Default value & Range of values used in the model \\
\hline$\mu$ & $\begin{array}{l}\text { Entry rate. To maintain a constant population size, the entry } \\
\text { and exit rates are assumed to be equal. }\end{array}$ & 0.02 per year & $\mathrm{n} / \mathrm{a}$ \\
\hline$\beta_{{ }^{\prime}}^{A} \beta_{k}^{B}$ & Transmission rate of strain $A$ or strain $B$ to sex $k$ per contact & 0.5 per contact & $0<\beta<1$ \\
\hline$\sigma_{k}^{A}, \sigma_{k}^{B}$ & $\begin{array}{l}\text { Recovery rate from asymptomatic infection with } A \text { or B per } \\
\text { year (recovery rate }=1 / \text { duration) } \\
\sigma=2 \text { per year }- \text { that is, average duration of } 6 \text { months } \\
\text { without treatment, decreasing to } 10 \text { per year ( } 1.2 \text { months } \\
\text { duration with treatment) }\end{array}$ & $\begin{array}{l}\sigma=2 \text { per year without treatment, } \\
\text { increasing to } 10 \text { per year, with treatment }\end{array}$ & $\begin{array}{l}\text { With treatment, recovery depends } \\
\text { on efficacy of treatment and the } \\
\text { average time taken to seek } \\
\text { treatment }\end{array}$ \\
\hline \multirow[t]{2}{*}{$\gamma^{A}, \gamma^{B}$} & \multirow{2}{*}{$\begin{array}{l}\text { Proportion of contacts with superinfected individuals, which } \\
\text { result in transmission of } A \text { or } B \text { only. (If } \gamma^{A}+\gamma^{B}=0 \text {, then all } \\
\text { contacts result in transmission of superinfection, if } \gamma^{A}+\gamma^{B}= \\
1, \text { then all contacts with coinfected individuals result in the } \\
\text { transmission of a single strain }\end{array}$} & $\gamma^{A}=\gamma^{B}=0.4$ & $0 \leqslant \gamma \geqslant 1$ \\
\hline & & $\begin{array}{l}\text { Therefore proportion of contacts which } \\
\text { result direct transmission of coinfection is: } \\
1-\left(\gamma^{4}+\gamma^{B}\right)=0.2\end{array}$ & $\gamma^{A}+\gamma^{B} \leqslant 1$ \\
\hline \multirow[t]{3}{*}{$\omega^{\mathrm{A}}, \omega^{\mathrm{B}}$} & $\begin{array}{l}\text { Proportion of those recovering from superinfection who } \\
\text { remain infected with strain } A \text { or } B \text {. The extreme cases are }\end{array}$ & $\omega^{\wedge}=\omega^{\mathrm{B}}=0.2$ & $0 \leqslant \omega \geqslant 0.5$ \\
\hline & $\begin{array}{l}\text { susceptible class }\left(\omega^{A}=\omega^{B}=0\right) \text {; or all recovery is from single } \\
\text { infections }\left(\omega^{A}+\omega^{B}=1\right)\end{array}$ & & $\omega^{\mathrm{A}}+\omega^{\mathrm{B}} \leqslant 1$ \\
\hline & $\begin{array}{l}\text { In the examples given in the text, } 40 \% \text { of recovery from } \\
\text { coinfection is from a single strain (equally split between the } \\
\text { two strains), and } 60 \% \text { is recovery to the susceptible class } \\
\text { directly, for example due to treatment }\end{array}$ & & \\
\hline$\phi^{A}, \phi^{B}$ & $\begin{array}{l}\text { Protective effect of infection with strain } A \text {, or strain } B \text { on } \\
\text { likelihood of transmission of different strains (total protective } \\
\text { effect, no coinfection }\left(\phi^{\wedge}=\phi^{B}=0\right) \text { to no protective effect }\left(\phi^{A}\right. \\
\left.=\phi^{B}=1\right) \text {. An enhancing effect of transmission due to prior } \\
\text { infection could also be given by } \phi^{A} \text { or } \phi^{B}>1\end{array}$ & $\phi^{A}=\phi^{B}=0.5$ & $\begin{array}{l}0 \leqslant \phi \leqslant 1 \text { for protective effect, or } \phi \\
>1 \text { for enhancing effect }\end{array}$ \\
\hline$\rho_{\mathrm{klm}}$ & $\begin{array}{l}\text { Mixing matrix, element } \mathrm{klm} \text { is the probability that individual } \\
\text { of sex } \mathrm{k} \text { in activity class I will form a partnership with a } \\
\text { member of activity class } \mathrm{m} \text { of the opposite sex }\end{array}$ & See appendix & $\mathrm{n} / \mathrm{a}$ \\
\hline$c_{k l}$ & $\begin{array}{l}\text { Contact rate of individual of sex k, activity class I (see } \\
\text { below) }\end{array}$ & See appendix & $n / a$ \\
\hline$\epsilon$ & $\begin{array}{l}\text { Pattern of mixing } \\
(0=\text { fully assortative, } 1=\text { random })\end{array}$ & 1 (random) & $0 \leqslant \epsilon \geqslant 1$ \\
\hline
\end{tabular}

opposite sex. The value of $\rho_{k \mid m}$ depends on the pattern of mixing and could vary from fully assortative, where partnerships only form within the same activity group to random, where partnerships form randomly between different activity class.

$\rho_{k l m}=\varepsilon \delta_{i j}+(1-\varepsilon) c_{k^{\prime} m} \frac{N_{k^{\prime} m}}{\sum_{u=1}^{4} c_{k^{\prime} u} N_{k^{\prime} u}}$

$\delta_{i j}=1$ when $\mathrm{i}=\mathrm{j}$ and $\delta_{\mathrm{ii}}=0$ when $\mathrm{i} \neq \mathrm{j}$.

It is necessary that the number of partnerships formed by sex $k$ match those of the other sex. For the sake of simplicity in these models both sexes are divided into classes of identical size and activity levels. While the models can include a range of mixing patterns between activity classes (from fully assortative to random) for simplicity we use a random pattern of mixing between different activity classes throughout, i.e. $(\epsilon=0)$.

Table 2 shows the contact rate parameters.

Model 2. Two strains, two sexes, four activity classes with asymptomatic and symptomatic infection.

Formulae to accompany two-strain model incorporating asymptomatic and symptomatic infections with no superinfection. Super-

\section{Table 2 Contact rate parameters}

\begin{tabular}{lll}
\hline Activity class & $\begin{array}{l}\text { Rate of partner } \\
\text { change (per year) }\end{array}$ & $\begin{array}{l}\text { Proportion of } \\
\text { population in class }\end{array}$ \\
\hline 1 & 40 & 0.02 \\
2 & 10 & 0.08 \\
3 & 2 & 0.3 \\
4 & 0.5 & 0.6 \\
\hline
\end{tabular}

scripts ' $a$ ' or ' $s$ ' denotes asymptomatic and symptomatically infected populations, respectively; for example, $\mathbf{A}_{\mathrm{kl}}^{\mathrm{a}}$ is the population infected with strain $\mathrm{E}$ who are asymptomatic. Capital superscripts indicate the strain as before.

Again we can define the force of infection $(\lambda)$, for strains A and B and we assume no coinfection:

$\lambda_{k l}^{A}=c_{k l} \beta_{k}^{A}\left(\psi^{A} \sum_{m=1}^{4} \rho_{k l m}\left(\frac{A_{k^{\prime} m}^{a}}{N_{k^{\prime} m}}\right)+\sum_{m=1}^{4} \rho_{k l m}\left(\frac{A_{k^{\prime} m}^{s}}{N_{k^{\prime} m}}\right)\right)$

$\lambda_{k l}^{B}=c_{k l} \beta_{k}^{B}\left(\psi^{B} \sum_{m=1}^{4} \rho_{k l m}\left(\frac{B_{k^{\prime} m}^{a}}{N_{k^{\prime} m}}\right)+\sum_{m=1}^{4} \rho_{k l m}\left(\frac{B_{k^{\prime} m}^{s}}{N_{k^{\prime} m}}\right)\right)$

The equations describing the model are as follows:

$$
\begin{aligned}
\frac{d X_{k l}}{d t}= & \mu N_{k l}-X_{k l}\left(\lambda_{k l}^{A}+\lambda_{k l}^{B}\right)+\sigma_{k}^{A a} A_{k l}^{a} \\
& +\sigma_{k}^{A s} A_{k l}^{S}+\sigma_{k}^{B a} B_{k l}^{a}+\sigma_{k}^{B s} B_{k l}^{S}-\mu X_{k l} \\
\frac{d A_{k l}^{a}}{d t}= & \theta^{A} X_{k l} \lambda_{k l}^{A}-\sigma_{k}^{A a} A_{k l}^{a}-\mu A_{k l}^{a} \\
\frac{d A_{k l}^{s}}{d t}= & \left(1-\theta^{A}\right) X_{k l} \lambda_{k l}^{A}-\sigma_{k}^{A s} A_{k l}^{s}-\mu A_{k l}^{s} \\
\frac{d B_{k l}^{a}}{d t}= & \theta^{B} X_{k l} \lambda_{k l}^{B}-\sigma_{k}^{B a} B_{k l}^{a}-\mu B_{k l}^{a} \\
\frac{d B_{k l}^{s}}{d t}= & \left(1-\theta^{B}\right) X_{k l} \lambda_{k l}^{B}-\sigma_{k}^{B s} B_{k l}^{s}-\mu B_{k l}^{s}
\end{aligned}
$$


Table 3 Additional parameters for asymptomatic model (model 2)

\begin{tabular}{|c|c|c|c|}
\hline Parameter & Definition & Default value & $\begin{array}{l}\text { Range of values used in } \\
\text { the model }\end{array}$ \\
\hline$\theta^{A}, \theta^{B}$ & $\begin{array}{l}\text { Percentage of infections with } A \text { or } B \\
\text { which are asymptomatic }\end{array}$ & $\theta^{A}=0.8 \theta^{B}=0.4$ & $\mathrm{n} / \mathrm{a}$ \\
\hline$\psi^{A}, \psi^{B}$ & $\begin{array}{l}\text { Transmissibility of asymptomatic } \\
\text { infections as a percentage of } \\
\text { symptomatic infections transmissibility. } \\
\text { (If } \psi=0 \text {, then only symptomatic } \\
\text { infections are transmitted, if } \psi=0.5 \text {, the } \\
\text { transmissibility of asymptomatic } \\
\text { infections is } 50 \% \text { that of symptomatic, } \\
\text { for } \psi=1 \text {, transmissibility of both } \\
\text { infections is the same. Potentially there } \\
\text { could be an increase in the relative } \\
\text { transmissibility of asymptomatic } \\
\text { infections, due to lack of awareness of } \\
\text { infections, and therefore no change in } \\
\text { sexual behaviour (e.g. condom use or } \\
\text { abstention). }\end{array}$ & $\begin{array}{l}1 \text { (No difference between } \\
\text { asymptomatic and } \\
\text { symptomatic infections) }\end{array}$ & $n / a$ \\
\hline$\sigma_{k,}^{A a} \sigma_{k}^{B a}$ & $\begin{array}{l}\text { Recovery rate from asymptomatic } \\
\text { infection with } \mathrm{A} \text { or } \mathrm{B} \text { per year } \\
\text { (recovery rate }=1 \text { /duration) }\end{array}$ & $\begin{array}{l}\sigma=2 \text { per year, that is, } \\
\text { average duration of } 6 \\
\text { months without treatment, } \\
\text { decreasing to } 10 \text { per year, } \\
\text { i.e. } 1.2 \text { months duration } \\
\text { with treatment }\end{array}$ & $\begin{array}{l}\text { With treatment, recovery } \\
\text { depends on efficacy of } \\
\text { treatment and the average } \\
\text { time taken to seek } \\
\text { treatment. With treatment, } \\
\text { recovery depends on } \\
\text { efficacy of treatment and } \\
\text { the average time taken to } \\
\text { seek treatment. With } \\
\text { treatment, recovery } \\
\text { depends on efficacy of } \\
\text { treatment and the average } \\
\text { time taken to seek treatment }\end{array}$ \\
\hline$\sigma_{k,}^{A s} \sigma_{k}^{B s}$ & $\begin{array}{l}\text { Recovery rate from symptomatic } \\
\text { infection with } A \text { or } B \text { per year } \\
\text { (recovery rate }=1 / \text { duration) }\end{array}$ & & \\
\hline
\end{tabular}

Additional parameters for an asymptomatic model are given in table 3 .

The models were solved numerically using a standard fourth order Runge-Kutta method.

\section{REFERENCES}

1 Wasserheit JN, Aral SO. The dynamic topology of sexually transmitted diseases: Implications for prevention strategies. J Infect Dis 1996;174(suppl 2):S201-13

2 Garnett GP. The geographic and temporal evolution of sexually transmitted disease epidemics. Sex Transm Infect 2002;78/Suppl I): i 14-19.

3 Anderson RM, May RM. Parasites, genetic variability and drug resistance. Infectious diseases of humans: dynamics and control. Oxford: Oxford University Press, 1991:607-25.

4 Mayr E. Animal species and evolution. Cambridge, MA: Harvard University Press, 1963.

5 Ward $\mathbf{H}$, Ison CA, Day SE, et al. A prospective social and molecular investigation of gonococcal transmission. Lancet 2000;365:1812-17.

6 Stephens RS, Wagar EA, Schoolnik GK. High resolution mapping of serovar-specific and common antigenic determinants of the major outer-membrane protein of Chlamydia trachomatis. J Exp Med 1988;167:817-31

7 Nowak MA, Anderson RM, Mclean AR, et al. Antigenic diversity threshold and the development of AIDS. Science 1991;254:963-9.

8 Schouls LM. Molecular biology of Treponema pallidum. In: Wright D, Archard L, eds. Molecular and cell biology of sexually transmitted diseases. London: Chapman and Hall, 1992:85-130.

9 Corey L, Wald A. Genital herpes. In: Holmes K, et al. Sexually transmitted diseases, 3rd ed. New York: McGraw-Hill, 1999:285-312.

10 Galloway DA. Biology of genital human papillomaviruses. In: Holmes K, et al. Sexually transmitted diseases, 3rd ed. New York: McGraw-Hill, 1999:335-46.

11 McKeown T. The origins of human disease. Oxford: Blackwell, 1988

12 Anderson RM, May RM. Co-evolution of hosts and parasites. Parasitology 1982;85:411-26.

13 Bull JJ. Perspectives - virulence. Evolution 1994;48:1423-37.

14 Lipsitch M. Bacterial population genetics and disease. Science 2001;292:59-60.

15 Lipsitch MA, Nowak M. The evolution of virulence in sexually transmitted HIV/AIDS. J Theor Biol 1995;174:427-40.
16 Lipsitch M, Moxon ER. Virulence and transmissibility of pathogens: what is the relationship? Trends Microbiol 1997:5:31-7.

17 Quetel C. History of syphilis. Cambridge: Polity Press, 1990.

18 Garnett GP, Aral SO, Hoyle DV, et al. The natural history of syphilis: its implications for the transmission dynamics and control of infection. Sex Transm Dis 1997;24:185-200.

19 Fenner F, Radcliffe FN. Myxomatosis. Cambridge: Cambridge University Press, 1965.

20 Gillespie SH. Antimicrobial resistance in the absence of selective pressure. Int J Antimicrob Agents 2001;17:171-6

21 Levin BR, Perrot V, Walker N. Compensatory mutations, antibiotic resistance and the population genetics of adaptive evolution in bacteria. Genetics 2000;154:985-97.

22 Haldane JBS. The cost of natural selection. J Genet 1957:55:51 1-24.

23 Kimura M. The neutral theory of molecular evolution. Cambridge: Cambridge University Press, 1983.

24 Hethcote HW, Yorke JA. Gonorrhea transmission, dynamics and control. Lecture notes in Biomathematics. Berlin: Springer Verlag, 1984. http://www/math.uiowa.edu/ftp/hethcote/Inb56.pdf

25 Anderson RM, May RM. The population biology of the interaction between HIV-1 and HIV-2: coexistence or competitive exclusion. AIDS 1996;10:1663-73.

26 Anderson RM. The Croonian Lecture, 1994. Populations, infectious disease and immunity: a very non-linear world. Phil Trans $R$ Soc Lond $B$ 1994;346:457-505

27 Condit R, Levin BR. The evolution of plasmid carrying multiple resistance genes - the role of segregation, transposition and homologous recombination. American Naturalist 1990;135:573-96.

28 Levin BR, Bergstrom CT. Bacteria are different. Proc Natl Acad Sci USA 2000:97:6981-5.

29 Phillips L. Beta-lactamase-producing, penicillin-resistant gonococcus Lancet. 1976;2:656-7.

30 Carson R, Tasker E, Houle B, et al. Tetracycline-resistant Neisseria gonorrhoeae - Georgia, Pennsylvania, New Hampshire. MMWR. 1985:34:563-4,569-70.

31 Fox KK, Knapp JS, Holmes KK, et al. Antimicrobial resistance in Neisseria gonorrhoeae in the United States, 1988-1994: the emergence of decreased susceptibility to the fluoroquinolones. J Infect Dis 1997:175: 1396-403.

32 Austin DJ, Anderson RM. Studies of antibiotic resistance within the patient, hospitals and the community using simple mathematical models. Phil Trans R Soc Lond 1999;354:721-38.

33 Lipsitch $M$, Levin BR. The population dynamics of antimicrobial chemotherapy. Antimicrob Agents Chemother 1997;41:363-73.

34 Stewart FM, Antia R, Levin BR, et al. The population genetics of antibiotic resistance. II. Analytic theory for sustained populations of bacteria in a community of hosts. Theoret Pop Biol 1998;53:152-65. 
35 Crawford C, Knapp JS, Hale J, et al. Asymptomatic gonorrhea in men cause by gonococci with unique nutritional requirements. Science 1977;196:1352-3

36 Whittington WLH, Holmes KK. Unique gonococcal phenotype associated with asymptomatic infection in men and with erroneous diagnosis of nongonococcal urethritis. J Infect Dis 2000;181:1044-8.

37 Martin IC. Diversity of opa genes of Neisseria gonorrhoeae. London: University of London, 2001. [PhD thesis.]
38 Website. Centers for Disease Control. http://www.cdc.gov/nchstp/ dstd/stats_trends/1999survrpt.htm

39 Ghani AC, Swinton J, Garnett GP. The role of sexual partnership networks in the epidemiology of gonorrhea. Sex Transm Dis 1997;24:45-55.

40 Morris $M$, Kretzschmar $M$. Concurrent partnerships and the spread of HIV. AIDS 1997;11:641-8.

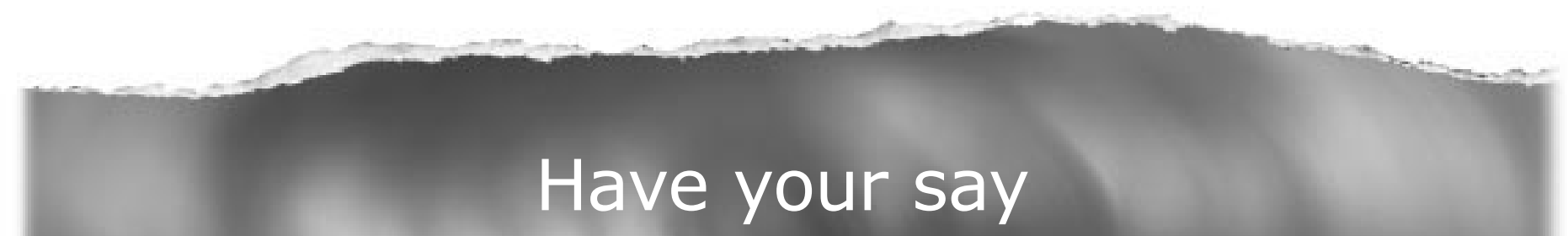

\section{eLetters}

If you wish to comment on any article published in Sexually Transmitted Infections you can send an eLetter using the eletters link at the beginning of each article. Your response will be posted on Sexually Transmitted Infections online within a few days of receipt (subject to editorial screening).

\section{www.sextransinf.com}

